\title{
Cardiac sarcoidosis
}

This article was published in the following Dove Press journal:

Research Reports in Clinical Cardiology

15 April 2016

Number of times this article has been viewed

\author{
Benedict T Costello ${ }^{1,2}$ \\ James $\mathrm{Nadel}^{3}$ \\ Andrew J Taylor ${ }^{1,2}$ \\ 'Department of Cardiovascular \\ Medicine, The Alfred Hospital, ${ }^{2}$ Baker \\ IDI Heart and Diabetes Research \\ Institute, Melbourne, VIC, ${ }^{3}$ School of \\ Medicine, University of Notre Dame, \\ Sydney, NSW, Australia
}

Correspondence: Andrew J Taylor Heart Centre, Third Floor Phillip Block, Commercial Road, Melbourne 3004,VIC, Australia

Tel +6I 390762363

Email a.taylor@alfred.org.au
Abstract: Cardiac sarcoidosis is a rare but life-threatening condition, requiring a high degree of clinical suspicion and low threshold for investigation to make the diagnosis. The cardiac manifestations include heart failure, conducting system disease, and arrhythmias predisposing to sudden cardiac death. A number of investigations are available to assist in making the diagnosis. The diagnosis may be made from the clinical history and evidence of inflammation on imaging modalities in the active phase and evidence of myocardial scarring in the chronic phase.

Keywords: cardiac magnetic resonance, positron emission tomography, sarcoidosis, sudden cardiac death

\section{Clinical vignette}

A 34-year-old man presented with fever following a period of overseas travel and underwent computed tomography of the chest. In this study, there were signs suspicious for pulmonary sarcoidosis with small bilateral noncalcified mediastinal and hilar lymph nodes, yet he was lost to follow-up. The following year, the same patient presented with acute shortness of breath following an aeroplane flight. Clinical examination revealed tachycardia (120 bpm), hypotension $(100 / 70 \mathrm{mmHg})$, and signs of congestive cardiac failure. An electrocardiogram showed evidence of conducting system disease with a first-degree heart block and left anterior hemiblock (Figure 1). The patient was referred for echocardiography (echo), which showed severe left ventricular (LV) systolic dysfunction, and an echodensity in the LV apex was suspicious for thrombus. An angiotensin-converting enzyme level was within normal limits, and serum calcium was slightly elevated at $2.53 \mathrm{mmol} / \mathrm{L}$ (normal range: $2.14-2.50 \mathrm{mmol} / \mathrm{L}$ ). Urinary calcium levels were also within normal limits.

He was referred for cardiac magnetic resonance (CMR) imaging, which confirmed severely reduced LV function and also demonstrated widespread late contrast enhancement in a noncoronary distribution (Figure 2). On the basis of these findings, the clinical diagnosis of cardiac sarcoidosis (CS) was highly likely. A positron emission tomography (PET) scan was also supportive of this diagnosis (Figure 3), with heterogeneous ${ }^{18} \mathrm{~F}$-fluorodeoxyglucose $\left({ }^{18} \mathrm{~F}\right.$-FDG) uptake corresponding to areas with late gadolinium enhancement (LGE) on CMR and reduced perfusion in the inflamed segments.

The patient was subsequently referred for cardiac biopsy. Despite an attempt to attain cardiac tissue from the areas of the heart corresponding to the contrast enhancement on the CMR images, there was no evidence of sarcoidosis on the cardiac biopsy specimens. However, an endobronchial biopsy was then performed, which demonstrated the 


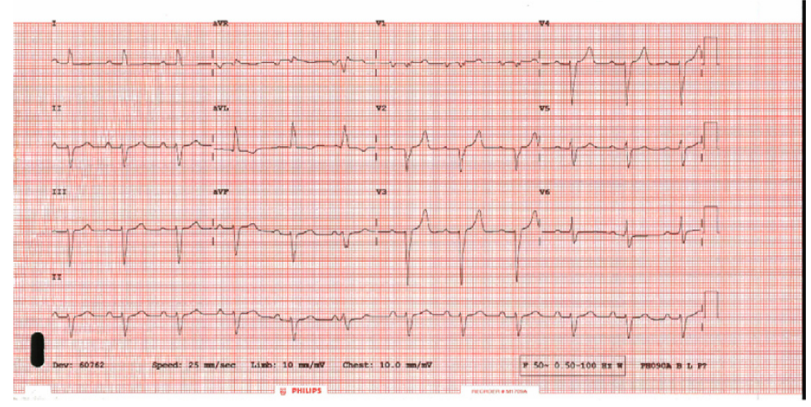

Figure I Electrocardiogram in a patient with CS demonstrating first-degree heart block and left anterior hemiblock.

Abbreviation: CS, cardiac sarcoidosis.

typical noncaseating granulomas consistent with sarcoidosis (Figure 4). The combination of this finding and the abnormalities on cardiac imaging allowed a histologic diagnosis of CS.

Based on the diagnosis and with a reduced ejection fraction, he was referred for implantation of an automatic implantable cardiovertor defibrillator (AICD). There has been no therapy from the AICD in the 6 months since it was implanted. At last clinical review, he was stable, breathless on moderate levels of exertion, and has had no significant change in ejection fraction despite initial aggressive steroid therapy.

Sarcoidosis is a multisystem granulomatous disorder of unclear etiology with a significant prevalence of cardiac involvement. CS can be a life-threatening condition, requiring a high level of clinical suspicion and a low threshold for investigation to make the diagnosis. The patient provided written informed consent for the use of the information and images in this review. The Alfred Hospital ethical review
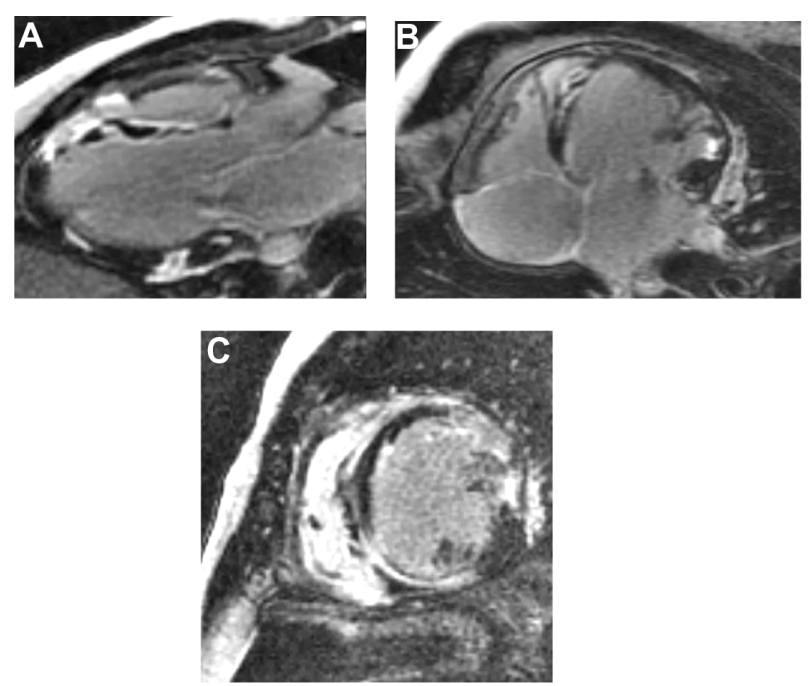

Figure 2 CMR images in a patient with CS, demonstrating the widespread contrast enhancement (LGE) typical of granuloma, extending throughout the myocardium and not constrained to a coronary artery distribution.

Notes: (A) Apical three-chamber view. (B) Apical four-chamber view. (C) Basal short axis view of the LV.

Abbreviations: CMR, cardiac magnetic resonance; CS, cardiac sarcoidosis; LGE, late gadolinium enhancement; LV, left ventricle.
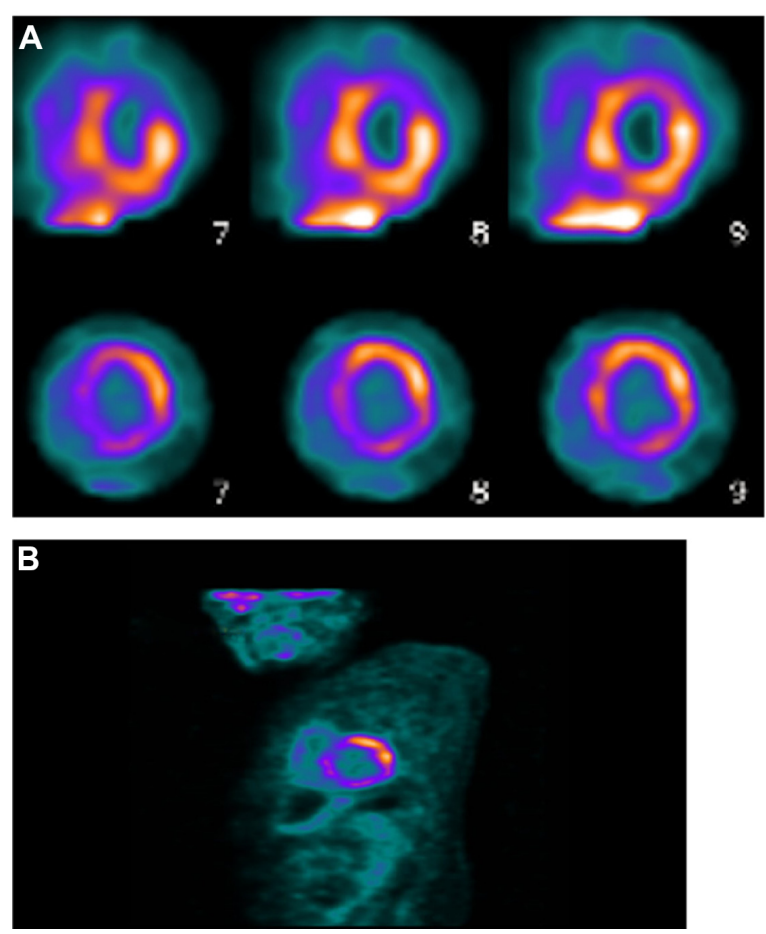

Figure 3 PET images of a patient with CS.

Notes: (A) PET image of left ventricle in short axis: resting perfusion imaging (top row) show defects in the anterolateral wall corresponding to areas of inflammation on ${ }^{18} \mathrm{~F}-\mathrm{FDG}$ images (bottom row). (B) Image taken from whole-body PET demonstrating heterogeneous ${ }^{18} \mathrm{~F}-\mathrm{FDG}$ uptake consistent with CS.

Abbreviations: PET, positron emission tomography; ${ }^{18} \mathrm{~F}-\mathrm{FDG},{ }^{18} \mathrm{~F}$-fluorodeoxyglucose; CS, cardiac sarcoidosis.

board deemed ethics approval unnecessary for this study under their Institutional guidelines, as patient images were anonymized and could not be traced back to patients.

\section{Incidence and pathophysiology Systemic sarcoidosis}

Systemic sarcoidosis is a disease characterized by noncaseating granulomas in involved organs (Figure 1). Sarcoidosis

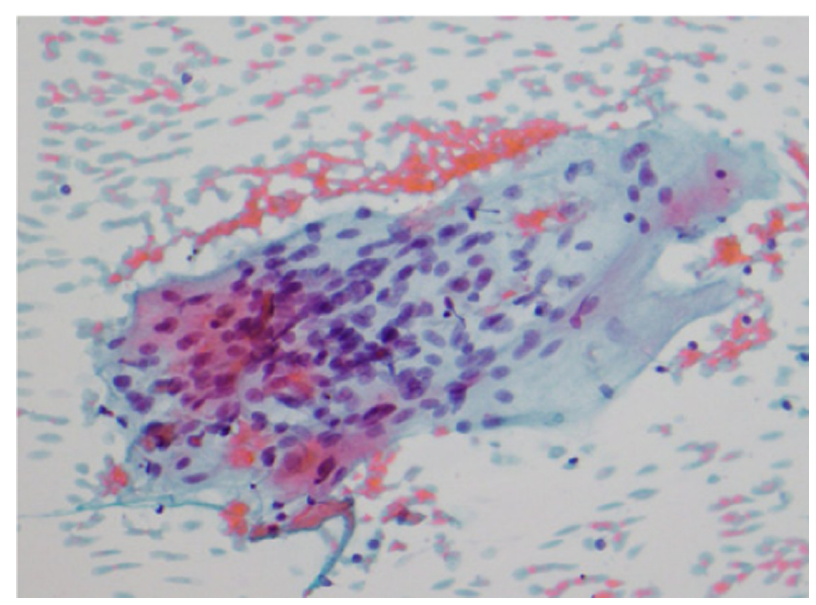

Figure 4 Endobronchial guided fine-needle aspirate of lymph node with clusters of epithelioid histiocytes with associated smeared lymphocytes, a feature of nonnecrotizing granulomas.

Note: Magnification is $400 \times$. 
commonly affects the lungs but can affect almost all organs. The development and accumulation of granulomas are fundamental to the pathophysiology of sarcoidosis. While the precise trigger for granuloma formation in sarcoidosis is unknown, granulomas generally have a role in isolating pathogens, restricting inflammation, and protecting surrounding tissues.

Sarcoidosis affects all racial and ethnic groups, with onset typically before the age of 50 years. The incidence varies widely across the globe, with the highest incidence in Northern Europe $(30 / 100,000) .{ }^{1}$ In the USA, the sarcoidosis incidence is three times higher in African-Americans than in the Caucasian population. ${ }^{2}$ The incidence in Australia is $\sim 5 / 100,000,{ }^{3}$ similar to the incidence in US Caucasians.

\section{Cardiac involvement}

Up to $50 \%$ of patients with pulmonary sarcoidosis have cardiac involvement; however, as few as 5\% will report cardiac symptoms. ${ }^{4}$ The initial presentation of CS is often sudden cardiac death (SCD), and so screening of patients with extra-CS for cardiac involvement is critical. ${ }^{5}$ Only 40\%-50\% of patients with CS diagnosed at autopsy have the diagnosis made during their lifetime. ${ }^{6}$

\section{Cardiac manifestations}

CS can manifest as complete heart block, ventricular arrhythmias, congestive heart failure, pericardial effusion, pulmonary hypertension, and ventricular aneurysms. ${ }^{7}$ These manifestations are a result of granuloma formation and inflammation within the myocardium, disrupting the structural integrity of the heart. In rare cases, CS will be the only manifestation of the disease ${ }^{8}$ Primary CS is a diagnostic challenge, with a high index of suspicion required in cases with unexpected reduction in cardiac function or rhythm disturbance. As a result, exclusive cardiac sarcoid may be first detected at autopsy.

\section{Diagnosis}

A variety of investigative modalities have been advocated for the detection of CS, including electrocardiogram (ECG), echo, radionucleotide studies (thallium-201 [ $\left.{ }^{201} \mathrm{Tl}\right]$ or technetium-99 [ $\left.{ }^{99 \mathrm{~m}} \mathrm{Tc}\right]$ scintigraphy), CMR, PET, and endomyocardial biopsy. The sensitivity and specificity vary between the tests ${ }^{9}$ (Table 1), and the choice of diagnostic modality depends on the availability, cost, and local expertise.

\section{Established algorithms for the diagnosis of CS}

A number of similar diagnostic algorithms have been proposed for the diagnosis of CS. ${ }^{10,11}$ Due to the lack of any true gold standard for the diagnosis of CS, no algorithm has
Table I Sensitivities and specificities of imaging modalities for the detection of cardiac sarcoidosis

\begin{tabular}{lll}
\hline Diagnostic modality & Sensitivity & Specificity \\
\hline ECG & Low & Low \\
Echo & Low-moderate & Low \\
${ }^{201} \mathrm{TI}$ or ${ }^{99 \mathrm{~m} T \mathrm{Tc}}$ & Moderate & Moderate \\
$\mathrm{CMR}$ & Moderate-high & High \\
$\mathrm{PET}$ & High & Moderate-high \\
EMB & Low-moderate & High \\
\hline
\end{tabular}

Note: Reprinted from Am Heart J, 157(I), Kim JS, Judson MA, Donnino R, et al, Cardiac sarcoidosis, 9-21, Copyright 2009, with permission from Elsevier. ${ }^{9}$

Abbreviations: ECG, electrocardiogram; Echo, echocardiography; ${ }^{201} \mathrm{Tl}$, thallium-20I;

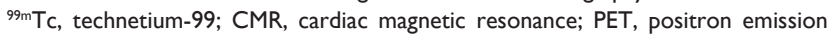
tomography; EMB, endomyocardial biopsy.

been adequately validated; as such the diagnosis is usually made through integration of clinical and imaging findings. The Japanese Ministry for Health (JMH) guidelines ${ }^{11}$ (1993, updated 2006) are widely cited; more recently, the Heart Rhythm Society (HRS) in 2014 proposed an algorithm for a definite or probable diagnosis of CS using biopsy and updated clinical criteria ${ }^{11}$ (Table 2). Another proposed diagnostic pathway is the National Institutes of Health's A Case Control Etiologic of Sarcoidosis Study, originally published in 1999 and updated in 2014 by the World Association for Sarcoidosis and Other Granulomatous Disorders (WASOG). ${ }^{12}$ Both the WASOG and HRS guidelines include CMR in the diagnostic algorithm and do not require histology for diagnosis. The WASOG criteria include a defect on perfusion scintigraphy or single-photon emission computed tomography scan, not present in the seven HRS criteria.

To summarize the algorithms, a diagnosis of CS can be reached in two ways. A definite diagnosis can be reached if a histologic specimen is consistent with sarcoidosis. A probable diagnosis can be reached if a combination of clinical, ECG, and imaging criteria is met; a probable diagnosis in this setting is sufficient to make a clinical diagnosis and initiate management strategies.

\section{Biopsy}

Myocardial biopsy can confirm the diagnosis of CS, provided the biopsy specimen contains areas of affected tissue. Imaging with CMR or PET may help identify areas of disease activity to guide the location of the biopsy. However, due to the focal nature of the disease, endomyocardial biopsy has a sensitivity as low as $30 \%$, often missing areas of cardiac involvement. ${ }^{13}$

\section{Biomarkers}

Biomarkers are nonspecific and have not been included in any diagnostic algorithms for CS. Biomarkers that may be elevated in CS include troponin (T or I), plasma B-type 
Table 2 Diagnostic algorithm adapted from the Heart Rhythm Society Guidelines

\begin{tabular}{ll}
\hline $\begin{array}{l}\text { Histological diagnosis } \\
\text { from myocardial tissue } \\
\text { (definite) }\end{array}$ & $\begin{array}{l}\text { Noncaseating granuloma on } \\
\text { myocardial biopsy without } \\
\text { alternative cause }\end{array}$ \\
\hline $\begin{array}{l}\text { Clinical diagnosis from } \\
\text { invasive and noninvasive }\end{array}$ & $\begin{array}{l}\text { Histologic diagnosis of extracardiac } \\
\text { studies (probable) }\end{array}$ \\
$\begin{array}{l}\text { One of: } \\
\text { - Steroid responsive } \\
\text { cardiomyopathy or heart block } \\
\text { - Unexplained EF }<40 \% \\
\text { - Unexplained sustained VT } \\
\text { - Advanced heart block } \\
\text { • Patchy uptake on cardiac PET } \\
\text { - LGE on CMR } \\
\text { - Positive gallium uptake } \\
\text { and } \\
\text { Other causes of cardiac } \\
\text { manifestations have been excluded }\end{array}$ \\
\hline
\end{tabular}

Note: Reprinted from Heart Rhythm, I I (7), Birnie DH, Sauer WH, Bogun F, et al, HRS expert consensus statement on the diagnosis and management of arrhythmias associated with cardiac sarcoidosis, 1304-1323, Copyright 2014, with permission from Elsevier."

Abbreviations: PET, positron emission tomography; LGE, late gadolinium enhancement; CMR, cardiac magnetic resonance; EF, ejection fraction; VT, ventricular tachycardia.

natriuretic peptide, angiotensin-converting enzyme, and urinary calcium levels. ${ }^{14}$

\section{Electrocardiography}

Atrial arrhythmias, ventricular arrhythmias, and the development of atrioventricular block are common manifestations of CS. ${ }^{5}$ Electrocardiographic abnormalities are common and present in up to $90 \%$ of patients with CS; however, a normal ECG does not exclude the diagnosis. Ambulatory ECG (Holter) monitoring may detect heart rhythm abnormalities, with ventricular ectopic beats a common finding. However, abnormal ECG or Holter findings have not been demonstrated to correlate malignant arrhythmias and have been unable to predict SCD. ${ }^{5}$

\section{Echocardiography}

Echo is a readily available diagnostic tool sensitive to cardiac abnormalities, though with low specificity for the detection of CS. Nonspecific findings include wall thinning (leading to aneurysm formation), focal or global hypokinesis, diastolic dysfunction, and pericardial effusion. There is some evidence to suggest that wall motion abnormalities in the basal septal wall are more common in CS when compared to idiopathic dilated cardiomyopathy. ${ }^{15}$

While echo findings in CS are nonspecific, echo is also not sensitive in the detection of early disease. It is possible that advanced echo parameters such as global longitudinal strain and strain rate may better detect early myocardial dysfunction due to CS, but again this lacks specificity.

\section{Positron emission tomography}

Cardiac PET imaging for CS requires a combination of myocardial perfusion and inflammation imaging. Abnormalities of myocardial perfusion and scarring are detected using PET radiotracers, ${ }^{12} \mathrm{~N}$ ammonia and rubidium-82, while inflammation is assessed using the PET radiotracer ${ }^{18} \mathrm{~F}-\mathrm{FDG}$. Both sets of images are acquired independently but interpreted together (Figure 3). PET is less suitable for the diagnosis of CS in patients with ischemic heart disease due to the confounding effects of coronary artery disease on abnormal perfusion images.

Patients require adequate preparation prior to scanning to convert metabolism from glucose to fatty acids. An 18or a 6-hour fast with fatty meal (free from carbohydrate) is recommended to suppress physiologic myocardial activity and ensure ${ }^{18} \mathrm{~F}$-FDG uptake in the myocardium corresponds to areas of inflammation. A heterogenous pattern of ${ }^{18} \mathrm{~F}-\mathrm{FDG}$ is more likely to be pathologic and is associated with CS. ${ }^{18} \mathrm{~F}-\mathrm{FDG}$ uptake will be present during the active phase of $\mathrm{CS}$ and will diminish as the inflammation and granulomata are replaced by fibrosis.

PET has the technical advantages of high spatial resolution, quantitative analysis, and low patient radiation exposure. There is some evidence that PET has the highest sensitivity, and therefore negative predictive value among the noninvasive imaging techniques for the diagnosis of CS..$^{15}$ The presence of focal perfusion defects and FDG uptake on cardiac PET also identifies patients at higher risk of death or ventricular tachycardia (VT). These findings may offer prognostic value beyond clinical criteria, the presence of extra-CS and LV ejection fraction. ${ }^{16}{ }^{18} \mathrm{~F}-\mathrm{FDG}$ uptake into areas of active inflammation presumed because CS has also been shown to diminish markedly or even disappear after commencement of steroid therapy. ${ }^{17}$

\section{Radionucleotide imaging}

${ }^{201} \mathrm{Tl}$ and ${ }^{99 \mathrm{~m}} \mathrm{Tc}$ can be used to detect myocardial abnormalities in CS. Abnormalities include moderate-to-severe focal resting defects in either a "fixed" or a "reverse redistribution" pattern. Granulomata and associated fibrosis are thought to contribute to the fixed defects, while focal reversible microvascular constriction in coronary arterioles around the granulomas is thought to be the mechanism of reverse redistribution. In this situation, the resting defect improves following stress. ${ }^{18}$ 
However, radionucleotide imaging has inferior sensitivity and specificity to CMR and PET and as such has not been included in the HRS algorithm for CS diagnosis.

\section{CMR imaging}

CMR is a sensitive imaging modality for the detection of preclinical and clinical CS. The excellent image quality and ability to characterize myocardium through detection of myocardial inflammation and fibrosis gives an advantage over echo. CMR also has the advantage over radionucleotide studies and PET by not exposing patients to ionizing radiation, a particular benefit when repeat imaging is anticipated. A limitation of CMR is the contraindication to imaging in the presence of non-magnetic resonance imaging-compatible cardiac pacemakers (permanent pacemakers) and defibrillators.

The following features allow the detection and diagnosis of CS on CMR: ${ }^{19}$

1. Restrictive LV filling pattern - the LV is typically nondilated and can have preserved systolic function and dilated atria.

2. Myocardial granulomata - granulomata with associated fibrosis are bright on LGE imaging relative to the surrounding myocardium. The areas of LGE have a characteristic spotty or frond-like appearance, do not follow a coronary artery distribution, and may respond to immunosuppressive therapy.

3. Focal edema - inflammation and edema can be detected with CMR using early gadolinium enhancement and short tau inversion recovery sequences. Inflammation and edema are suggestive of active disease.

4. LV dysfunction - reduction in systolic function is not uncommon and the presence of ventricular aneurysms can be readily detected on CMR.

5. Extracardiac findings - typical pulmonary manifestations of sarcoidosis can be readily detected on magnetic resonance imaging. ${ }^{20}$ Pulmonary and hilar nodules as small as 3-4 mm may be detected. Lung involvement may progress to fibrosis, and frank fibrosis can be readily detected.

LGE does not discriminate between active, inflammatory disease and the chronic, fibrotic stage; however, when CMR is used in conjunction with PET imaging, staging of the disease may be possible, as outlined in Table 3 .

A number of prior studies have investigated whether the presence of LGE imaging on CMR can serve as a reliable indicator of sarcoid infiltration of the heart. LGE imaging has a high diagnostic accuracy in patients with presumed CS based on JMH criteria, with a sensitivity of $100 \%$ and a specificity of $78 \% .{ }^{21}$ Furthermore, when CMR imaging is
Table 3 Stages of disease activity and imaging findings

\begin{tabular}{llll}
\hline Stages & PET & & CMR \\
\cline { 2 - 4 } & $\begin{array}{l}\text { Perfusion } \\
\text { defect }\end{array}$ & $\begin{array}{l}\text { FDG } \\
\text { uptake }\end{array}$ & $\begin{array}{l}\text { LGE } \\
\text { enhancement }\end{array}$ \\
\hline Normal & None & Low & None \\
Early & Minimal & High & Minimal \\
Peak active & Severe & High & High \\
Chronic (fibrosis) & Severe & None & High \\
\hline
\end{tabular}

Abbreviations: PET, positron emission tomography; CMR, cardiac magnetic resonance; LGE, late gadolinium enhancement; FDG, fluorodeoxyglucose.

directly compared to JMH guidelines, the presence of LGE predicts twice as many longer term cardiac events than the JMH guidelines but with a similar false-positive rate, suggesting that LGE imaging may be significantly more sensitive than the JMH guidelines for the diagnosis of CS.22 Two recent studies suggested that the presence of myocardial scar indicated by LGE was the best independent predictor of SCD in patients with suspected CS. ${ }^{23}$

CMR may also be used to monitor response to treatment with immunosuppressive medications. A reduction in early contrast enhancement has been demonstrated on CMR following treatment ${ }^{24}$ and may represent a reduction in inflammation associated with acute CS. Another small study found that patients who clinically improved following treatment with corticosteroid had a reduction in LGE (scar) burden at 12-month follow-up..$^{25}$

However, it should be noted that the cardiac findings on CMR are not necessarily specific to CS and that the findings should be considered within the context of the clinical presentation and medical history. The differential includes myocarditis, ischemic heart disease, hypertrophic cardiomyopathy, and arrhythmogenic right ventricular dysplasia (ARVC).

\section{Distinguishing CS from ARVC}

$\mathrm{CS}$ and ARVC can have similar clinical manifestations, and patients with CS may satisfy diagnostic criteria for ARVC. A correct diagnosis is important for both management and familial screening, which differ between the conditions. CMR provides useful information for distinguishing between the two conditions, as patients with CS are more likely to have LV dysfunction and mediastinal lymphadenopathy, with septal fibrosis detected with delayed gadolinium enhancement. ${ }^{26}$

\section{Prognosis and treatment}

While the prognosis of asymptomatic CS is not well understood (due to the fact that, in many, this diagnosis may 
first be made at autopsy), the presence of LGE on CMR is associated with a high risk of SCD regardless of the presence of cardiac symptoms. ${ }^{22,23,27}$ Prior to the use of AICD therapy, SCD occurred in up to $65 \%,{ }^{9}$ often as the initial presentation. Five-year survival is currently estimated at up to $60 \%{ }^{7}$ The cause of death in CS is usually malignant arrhythmia and SCD or less commonly due to heart failure. ${ }^{15}$

Patients with evidence of CS on CMR have higher rates of adverse cardiovascular events than those with only extracardiac disease. ${ }^{27}$ In particular, patients with CS have a much higher rate of arrhythmia and SCD than those with extracardiac sarcoid. Importantly, in patients with CS diagnosed by LGE on CMR, those with an AICD/permanent pacemaker were less likely to experience SCD than those without, supporting the role of these devices in the management of CS. ${ }^{27}$

Similar to other rare conditions, treatment decisions are largely based on clinical experience and expert consensus rather than randomized trial data. Treatment of CS is based on the reduction of active disease with steroids and immunosuppression and prevention of SCD with AICDs.

Active disease can be detected using CMR and PET. The goal of the treatment is to suppress granuloma formation and reduce inflammation, reducing the development of myocardial scarring. There is evidence of a reduction in the clinical and radiological evidence of CS with immunosuppression, without a clear mortality benefit. ${ }^{17,28}$

In the chronic phase of CS, the disease progresses to a fibrotic stage and generates scar. As in patients with other cardiomyopathies, scar serves as a substrate for reentry, increasing the risk of VT and $\mathrm{SCD},{ }^{29}$ and contributes to the development of conducting system disease. As such, many patients are treated with implanted devices for primary or secondary prevention and high-degree atrioventricular block.

There are few data specific to ICD use in the CS population. There is a class IIa recommendation in the general device guidelines ${ }^{30}$ with the following wording: "ICD implantation is reasonable for patients with $\mathrm{CS}$, giant cell myocarditis, or Chagas disease". The recommendation is stronger in patients with suspected CS who have reduced ejection fraction or frequent ventricular arrhythmia. AICD may be useful following a positive electrophysiologic study in patients with CS who has LGE on CMR. ${ }^{31}$

Antiarrhythmic therapy or ablation may be useful in patients with ventricular arrhythmias, particularly in the absence of active inflammation. ${ }^{5}$ Patients with refractory heart failure or arrhythmias may be referred for transplantation.

\section{Conclusion}

$\mathrm{CS}$ is an underrecognized condition occurring in patients with systemic sarcoidosis. While establishing the diagnosis can be challenging, the high rate of cardiac failure and ventricular arrhythmias makes accurate diagnosis important, and this can be achieved with a combination of clinical vigilance and low threshold for investigation. There are emerging data to support the use of prophylactic AICD implantation in selected patients to prevent SCD - a catastrophic complication of CS; therefore, the identification of patients with CS is a vital goal of clinical care.

\section{Disclosure}

The authors report no conflicts of interest in this work.

\section{References}

1. Iannuzzi MC, Rybicki BA, Teirstein AS. Sarcoidosis. $N$ Engl $J$ Med. 2007;357(21):2153-2165.

2. American Thoracic Society. Statement on sarcoidosis. Am J Respir Crit Care Med. 1999;160(2):736-755.

3. Gillman A, Steinfort C. Sarcoidosis in Australia. Intern Med J. 2007;37(6):356-359.

4. Cheong BYC, Muthupillai R, Nemeth M, et al. The utility of delayed-enhancement magnetic resonance imaging for identifying nonischemic myocardial fibrosis in asymptomatic patients with biopsy-proven systemic sarcoidosis. Sarcoidosis Vasc Diffuse Lung Dis. 2009;26(1):39-46.

5. Zipse MM, Sauer WH. Electrophysiologic manifestations of cardiac sarcoidosis. Curr Opin Pulm Med. 2013;19(5):485-492.

6. Sekhri V, Sanal S, DeLorenzo LJ, et al. Cardiac sarcoidosis: a comprehensive review. Arch Med Sci. 2011;4:546-554.

7. Zipse MM, Sauer WH. Cardiac sarcoidosis. Curr Cardiol Rep. 2014;16(8):1-10.

8. Kandolin R, Lehtonen J, Graner M, et al. Diagnosing isolated cardiac sarcoidosis. J Intern Med. 2011;270(5):461-468.

9. Kim JS, Judson MA, Donnino R, et al. Cardiac sarcoidosis. Am Heart J. 2009;157(1):9-21.

10. Freeman AM, Curran-Everett D, Weinberger HD, et al. Predictors of cardiac sarcoidosis using commonly available cardiac studies. $\mathrm{Am} \mathrm{J}$ Cardiol. 2013;112(2):280-285.

11. Birnie DH, Sauer WH, Bogun F, et al. HRS expert consensus statement on the diagnosis and management of arrhythmias associated with cardiac sarcoidosis. Heart Rhythm. 2014;11(7):1304-1323.

12. Judson MA, Costabel U, Drent M, et al. The WASOG sarcoidosis organ assessment instrument: an update of a previous clinical tool. Sarcoidosis Vasc Diffuse Lung Dis. 2014;31(1):19-27.

13. Cooper LT, Baughman KL, Feldman AM, et al. The role of endomyocardial biopsy in the management of cardiovascular disease: a scientific statement from the American Heart Association, the American College of Cardiology, and the European Society of Cardiology endorsed by the Heart Failure Society of America and the Heart Failure Association of the European Society of Cardiology. J Am Coll Cardiol. 2007;50(19):1914-1931.

14. Aggarwal NR, Snipelisky D, Young PM, et al. Advances in imaging for diagnosis and management of cardiac sarcoidosis. Eur Heart $J$ Cardiovasc Imaging. 2015; pii:jev142.

15. Yazaki Y, Isobe M, Hiramitsu S, et al. Comparison of clinical features and prognosis of cardiac sarcoidosis and idiopathic dilated cardiomyopathy. Am J Cardiol. 1998;82(4):537-540.

16. Blankstein R, Osborne M, Naya M, et al. Cardiac positron emission tomography enhances prognostic assessments of patients with suspected cardiac sarcoidosis. J Am Coll Cardiol. 2014;63(4):329-336. 
17. Yamagishi H, Shirai N, Takagi M, et al. Identification of cardiac sarcoidosis with 13N-NH3/18F-FDG PET. J Nucl Med 2003;44(7):1030-1036.

18. Bulkley BH. The use of 201 thallium for myocardial perfusion imaging in sarcoid heart disease. Chest. 1977;72(1):27.

19. Kidambi A. CMR Pocket Guide. European Society of Cardiology; 2012:1-80. Leeds, UK.

20. Chung JH, Little BP, Forssen AV, et al. Proton MRI in the evaluation of pulmonary sarcoidosis: comparison to chest CT. Eur J Radiol. 2013;82(12):2378-2385.

21. Smedema J-P, Snoep G, van Kroonenburgh MPG, et al. Evaluation of the accuracy of gadolinium-enhanced cardiovascular magnetic resonance in the diagnosis of cardiac sarcoidosis. J Am Coll Cardiol. 2005;45(10):1683-1690.

22. Patel MR, Cawley PJ, Heitner JF, et al. Detection of myocardial damage in patients with sarcoidosis. Circulation. 2009;120(20):1969-1977.

23. Greulich S, Deluigi CC, Gloekler S, et al. CMR imaging predicts death and other adverse events in suspected cardiac sarcoidosis. JACC Cardiovasc Imaging. 2013;6(4):501-511.

24. Schulz-Menger J. Patterns of myocardial inflammation and scarring in sarcoidosis as assessed by cardiovascular magnetic resonance. Heart. 2005;92(3):399-400.

25. Vignaux O, Dhote R, Duboc D, et al. Clinical significance of myocardial magnetic resonance abnormalities in patients with sarcoidosis: a 1-year follow-up study. Chest. 2002;122(6):1895-1901.
26. Philips B, Madhavan S, James CA, et al. Arrhythmogenic right ventricular dysplasia/cardiomyopathy and cardiac sarcoidosis distinguishing features when the diagnosis is unclear. Circ Arrhythm Electrophysiol. 2014;7(2):230-236.

27. Nadel J, Lancefield T, Voskoboinik A, et al. Late gadolinium enhancement identified with cardiac magnetic resonance imaging in sarcoidosis patients is associated with long-term ventricular arrhythmia and sudden cardiac death. Eur Heart J Cardiovasc Imaging. 2015;16(6):634-641.

28. Ishikawa T. Steroid therapy in cardiac sarcoidosis. Increased left ventricular contractility concomitant with electrocardiographic improvement after prednisolone. Chest. 1984;85(3):445.

29. Furushima H, Chinushi M, Sugiura H, et al. Ventricular tachyarrhythmia associated with cardiac sarcoidosis: its mechanisms and outcome. Clin Cardiol. 2006;27(4):217-222.

30. Epstein AE, DiMarco JP, Ellenbogen KA, et al. ACC/AHA/HRS 2008 guidelines for device-based therapy of cardiac rhythm abnormalities. J Am Coll Cardiol. 2008;51(21):e1-e62.

31. Aizer A, Stern EH, Gomes JA, et al. Usefulness of programmed ventricular stimulation in predicting future arrhythmic events in patients with cardiac sarcoidosis. Am J Cardiol. 2005;96(2):276-282.
Research Reports in Clinical Cardiology

\section{Publish your work in this journal}

Research Reports in Clinical Cardiology is an international, peerreviewed, open access journal publishing original research, reports, editorials, reviews and commentaries on all areas of cardiology in the clinic and laboratory. The manuscript management system is completely online and includes a very quick and fair peer-review system.

\section{Dovepress}

Visit http://www.dovepress.com/testimonials.php to read real quotes from published authors. 